\title{
Hyperchloremic metabolic acidosis in the kidney transplant patient
}

Debora Avila-Poletti ${ }^{1}$, Leticia De Azevedo ${ }^{1}$, Candela lommi ${ }^{1}$ Kristian Heldal, MD. PhD. ${ }^{2}$, Carlos G. Musso, MD. PhD1

Human Physiology Department. Instituto Universitario del Hospital Italiano de Buenos Aires ${ }^{1}$

Clinic of Internal Medicine, Telemark Hospital Trust, Skien and Institute of Clinical Medicine, Faculty of Medicine of University of Oslo. Norway²

Running head: Hyperchloremic metabolic acidosis in kidney transplant

Key words: renal tubular acidosis, kidney transplantation, pathophysiology

\begin{abstract}
:
Hyperchloremic metabolic acidosis of renal origin results from a defect in renal tubular acidification mechanism, and this tubular dysfunction can consist of an altered tubular proton secretion or bicarbonate reabsorption capability. Studies have documented that all forms of renal tubular acidosis (RTA), type I to IV, are documented in kidney transplant patients. Among RTA pathophysiologic mechanisms have been described such as renal mass reduction, hyperkalemia, hyperparathyroidism, graft rejection, immunologic diseases, and some drugs such as renin-angiotensin-aldosterone blockers, and calcineurin inhibitors. RTA can lead to serious complications as is the case of muscle protein catabolism, muscle protein synthesis inhibition, renal osteodystrophy, renal damage progression, and anemia promotion. RTA should be treated by suppressing its etiologic factor (if it is possible), avoiding hyperkalemia, and/or supplying bicarbonate or a precursor (citrate). In conclusion: Hyperchloremic metabolic acidosis of renal origin is a relatively frequent complication in kidney transplantation patients, which can be harmful, and should be adequately treated in order to avoid its renal and systemic adverse effects.
\end{abstract}




\section{Introduction}

Metabolic acidosis, and particularly hyperchloremic metabolic acidosis (HMA) or metabolic acidosis with normal serum anion gag $(8-16 \mathrm{mmol} / \mathrm{L})$ is a frequent complication of chronic kidney disease (CKD) patients of whom glomerular filtration rate (GFR) decreases below $50 \mathrm{ml} / \mathrm{min} / 1.73 \mathrm{~m}^{2}$ (1). Even though, the prevalence of metabolic acidosis is higher in CKD patients than in kidney transplant recipients, the prevalence of this disorder ranges from $11 \%$ up to over $50 \%$ in kidney transplant patients (1-3). Experimental and clinical studies suggest that chronic metabolic acidosis could increase renal disease progression, and even morbidity and mortality in CKD patients (1). However, most of these studies have been performed in different stages of CKD, but not in kidney transplant recipients $(3,4)$. Even though HMA can be induced by intestinal bicarbonate loss in kidney transplant patients (eg: mycophenolate induced diarrhea), HMA is usually induced by renal tubular incapability to eliminate the hydrogen ion load derived by both dietary and endogenous sources and to generate bases (3).

The main difference between congenital renal tubular acidosis (RTA) and porttransplantation RTA is that the former characteristically involves dysfunction of specific transporter proteins or enzymes; while the latter is influenced by donor's kidney conditions, and/or inflammatory, ischemic, toxic events which involve the graft immediately before surgery or during the post-transplantation period (5-7).

Studies have documented that kidney transplant associated RTA is related to patient's age, transplantation period, graft function, hyperparathyroidism, hemoglobin level and treatment with angiotensin conversing enzyme inhibitors (ACEI), angiotensin receptor antagonists (ARA), and calcineurin inhibitors (CNI) (5-9). Even though, it has been reported that HMA is significantly higher in cadaveric donor recipients, there is as yet no reliable information on the impact of the donor renal function and cold ischaemia duration on the occurrence of post-kidney transplant metabolic acidosis (3).

Therefore, kidney transplant recipients are more likely to suffer from secondary RTA due to tubular dysfunction leading to a defect in $\mathrm{H}+$ excretion, bicarbonate reabsorption or both, usually inducing an asymptomatic $\operatorname{HMA}(8,9)$.

It is worth mentioning that primary RTA are infrequent entities (exact prevalence is unknown) which are considered as orphan diseases, their respective numbers are: proximal tubular acidosis ORPHA: 47159, and distal tubular acidosis ORPHA: 18. $(10,11)$ Among the main type of RTA described in kidney transplant patients are the following (Tabe1): 
- Type I distal RTA:

This tubular dysfunction has been associated to interstitial damage caused by acute or chronic transplant rejection, longterm intake of calcineurin inhibitors (CNI), or persistent (pre-transplant) or new (post-transplant) inflammatory status. In addition, hyperkalemic distal RTA (voltage dependent RTA) can be secondary to obstructive uropathy, since urinary tract obstruction can alter the normal negative tubular lumen voltage which is crucial for stimulating proton secretion (5,9-14).

-Type II (proximal) RTA:

This tubular disorder is characterized by the decrement of bicarbonate reabsorption in the proximal tubule because of the toxic effects of CNIs or post-transplant persisting hyperparathyroidism. RTA might completely improve during the first 6 months after transplantation since tubular damage and hyperparathyroidism resolves. This RTA can also be documented not only as an isolated entity but also joined to a complete proximal tubular deficiency (Fanconi syndrome) secondary to administered drugs or tubular ischemia usually found in post-transplant setting. Among the main Fanconi syndrome inducing drugs are: cisplatin, aminoglycosides, valproate, 6-mercaptopurine, and ifosfamide $(4,5,9,12)$.

-Type IV RTA:

This tubular functional alteration is mainly caused by the renin-angiotensin-aldosterone system (RAAS) suppressing drugs, trimethoprim-sulfamethoxazole, calcineurin inhibitors (CNI), adrenal insufficiency or hyporeninemic hypoaldosteronism secondary to diabetic, lupic or acquired immunodeficiency syndrome nephropathy. Less frequently, it can be induced by acute transplant rejection (3,5,8-16).

\section{RTA inducing mechanisms in kidney transplantation}

Several RTA inducing mechanisms in kidney transplant recipients have been described in the literature (5,9-13).

A) Reduced renal function (3-5,8-17):

This pathophysilogical mechanism results in a proportional impairment of both processes: acid excretion and base reclamation/regeneration, leading to a decreased renal net acid excretion of ammonium and titratable acidity, rather than a selective impairment of a specific tubule segment. CKD patients usually present HMA when their GFR is below 30 $\mathrm{ml} / \mathrm{min} / 1.73 \mathrm{~m}^{2}$ when the compensatory mechanisms of the residual functioning renal mass are usually not enough to adequately eliminate net acidity. However, this form of HMA is observed in kidney transplant patients when the graft function is well over the 
above-mentioned GFR threshold. To explain this phenomenon, it has been postulated that since a renal graft is a single kidney, it receives higher blood flow, and consequently impairs the transtubular electro-chemical gradient, reducing luminal negativity, and consequently affecting proton secretion, and bicarbonate reabsorption.

B) Insulin Resistance (3,12-26):

Insulin stimulates sodium/proton exchange in proximal tubular cells, which in turn induces urine ammonium excretion, and consequently insulin will stimulate proximal tubular ammoniagenesis. In accordance with these findings, hyperinsulinemia increases urinary ammonium excretion in humans. On the other hand, insulin resistance has been documented in renal transplant patients, which can be attributed to immunosuppressive drugs, especially corticosteroids and $\mathrm{CNI}$, as well as to an inflammatory status, and increased body fat mass. Thus, the combined effects of immunosuppressive therapy and metabolic changes after transplantation with development of insulin resistance may cause a renal acidification defect due to impaired renal ammoniagenesis. This disturbance in renal ammonia synthesis and acid excretion could be corrected by insulin sensitizers (eg: thiazolidinediones) which can improve insulin sensitivity in renal transplant patients. However, since not all individuals who suffer from insulin resistance present RTA, unknown factors should be implicated in its appearance.

C) Hyperkalemia $(3,9,12,27,28)$ :

A highly significant inverse linear relationship exists between serum potassium and whole blood base excess in kidney transplant recipients. Moreover, it has been proposed that HMA can be caused by hyperkalemia by at least two mechanisms:

Firstly, hyperkalemia can induce unfavorable (relatively positive) tubular lumen voltage, which impairs urine hydrogen secretion. Secondly, hyperkalemia induces potassium entry into the intracellular compartment in exchange with proton, which leads to intracellular alkalosis, and ammoniagenesis inhibition (29). It is worth mentioning that $\mathrm{CNI}$ can inhibit potassium secretion at the collecting duct by inhibiting basolateral sodium-potassiumATPase pump. This phenomenon results in a decline in sodium uptake impairing the transepithelial potassium secretion, consequently leading to hyperkalemia.

D) Calcineurin inhibitors (CNI) $(3,5,8-14,18,20,30)$ :

$\mathrm{CNI}$ are the most likely contributors to RTA in kidney transplant recipients among all the immunosuppressive drugs. As described above, $\mathrm{CNI}$ contribute via both the insulin resistance and hyperkalemia RTA inducing mechanisms. In addition, CNI can induce RTA by exerting structural damage (direct tubular toxic effect), and functional alteration 
(electrolyte tubular transport modification). CNI can increase sodium transporter activity, limiting sodium delivery to collecting tubules, and consequently altering distal tubular acidification. Blankenstein et al documented that direct epithelial action of CNI is sufficient for the $\mathrm{Na}-\mathrm{Cl}$ cotransporter activation, whereas its effect on furosemide-sensitive $\mathrm{Na}-\mathrm{K}-2 \mathrm{Cl}$ cotransporter requires concomitant vasopressin stimulation (30).

$\mathrm{CNI}$ inducing RTA effect depends on the drug dose, and a reduction of the dosage has been reported to be associated with a recovery of the RTA $(5,8-14,18)$.

It has been demonstrated that CNI nephrotoxicity can be seen as early as 3 months after kidney transplantation and it increases to $60-70 \%$ in 2 years. Thus, chronic CNI toxicity plays an important role in the development of RTA in long term kidney transplant recipients. It is known that acidosis induces transformation of beta-intercalated tubular cells, which secrete bicarbonate, into alfa-intercalated tubular cells, which secrete hydrogen. However, it has been documented that this phenomenon is inhibited by cyclosporine but not by tacrolimus (3). Inhibition of the intercalated cell transformation prevents an increased hydrogen secretion after an acid load, since the number of betaintercalated cells remains unchanged, bicarbonate excretion continues at the same rate leading to a net bicarbonate loss which results in a hyperchloremic metabolic acidosis with high urinary $\mathrm{pH}$ (classic RTA). Even though tacrolimus did not influence intercalated cell transformation, it has a more pronounced effect on reducing tubular $\mathrm{H}+$ secretion than cyclosporine, thus tacrolimus also induces distal RTA by a different mechanism. Even though, it has been reported that tacrolimus has a more pronounced effect on the development of distal RTA than cyclosporine, this finding has not been supported by all studies (3).

Finally, it is worth mentioning that CNI-induced hyperkalemic RTA in kidney transplant recipients can be managed by dosage reduction, adding low dose fludrocortisone, or transitory switching to sirolimus (31).

- Other causes:

Since RTA can appear in several immune-mediated diseases (eg: systemic erythematosus lupus, Sjôgren disease, hypergammaglobulinemic conditions), these disorders can also reappear in the kidney graft $(3,5,18,32,33)$.

Despite the fact that recent studies were not able to confirm a significant association between acute kidney rejection and RTA, few reports support the existence of this HMA inducing mechanism, and its neutralization after controlling kidney rejection with immunosuppressive treatment (3). 
A high prevalence of distal RTA has been documented in the transplant recipients receiving the mTOR inhibitor sirolimus, suggesting that this immunosuppressant may also cause distal tubular dysfunction. However, it is known if sirolimus can cause proteinuria and hypokalemia, the mechanism of development and prevalence of RTA in these patients remain unclear $(3,34)$.

Renin-angiotensin-aldosterone system (RAAS) inhibitors have been described to induce approximately $25 \%$ of cases with distal RTA in kidney transplant recipients $(3,32)$.

Hyperparathyroidism can also induce RTA by reducing the proximal tubule reabsorption capability (4). Finally, various explanations, including azathioprine toxicity and antigenantibody reaction, have been proposed to explain post-transplantation tubular acidification dysfunction $(3,10,28)$.

\section{Acidosis adverse effects}

Clinical and experimental studies have demonstrated that chronic metabolic acidosis secondary to RTA can lead to the following complications $(3,10)$.

- Muscle protein catabolism and inhibition of muscle protein synthesis $(3,10)$ :

Low serum bicarbonate concentration is associated with higher serum cortisol level, and the rate of protein degradation has been shown to be directly related to serum cortisol level and indirectly to serum bicarbonate level. Besides, acidosis related lean mass reduction can also be induced by insulin resistance.

- Renal osteodystrophy (3):

Bone mineral composition can be affected in a setting of metabolic acidosis since there is a reduction in bone formation and resorption, and growth retardation in children. Chronic metabolic acidosis reduces 1.25 vitamin $\mathrm{D}$ synthesis by proximal tubule, and may thereby limit calcium absorption from diet. Since chronic metabolic acidosis alters the homeostatic relationships between blood ionized calcium, parathyroid hormone, and active vitamin $D$, these patients are more vulnerable to suffer from osteopenia, and bone fractures, which is further worsened by the use of corticosteroids.

- Renal damage $(3,5,10,19)$ :

Metabolic acidosis can affect graft function by causing progressive tubulointerstitial injury. Administration of bicarbonate to individuals with CKD and metabolic acidosis slowed the progression of CKD. The decline in GFR was less than half of the control group who received sodium chloride. In addition, metabolic acidosis can promote nephrocalcinosis, and/or nephrolithiasis. 
- Excessive phosphaturia (3):

It has been documented that more than $90 \%$ of transplant recipients exhibit inappropriate phosphaturia during the first months post-transplant. Moreover, metabolic acidosis is associated with an increase in urinary phosphate loss, since metabolic acidosis induces phosphaturia by impairing renal proximal tubular apical sodium gradient-dependent phosphate transporter activity. It is worth pointing out that phosphaturia induced by metabolic acidosis is independent of endogenous parathyroid hormone activity. In metabolic acidosis the increase in urinary phosphate may contribute substantially to renal proton secretion by increasing titratable acidity. Thus, phosphaturia can compensate the impaired renal net acid excretion in transplant patients. However, this hyperphosphaturia can also lead to muscle phosphorous depletion.

- Acidic environment could contribute to induce anemia in this population $(11,12,19)$.

\section{RTA Treatment}

Acid-base status should be monthly monitored in all kidney transplant patients in order to early detect and treat RTA in this population.

The basis of HMA treatment consists of administering appropriate amounts of alkali in the form of either bicarbonate or citrate. The amount of alkali administered should compensate for the urinary loss of bicarbonate plus the amount of acid generated by the protein catabolism. Besides, it has been strongly proposed that alkalitherapy represents a welltolerated, safe and cost-effective treatment which can slow the progression towards graft failure, therefore prolonging long-term graft survival (34). In clinical practice, metabolic acidosis is usually corrected when serum bicarbonate level is $<22 \mathrm{mmol} / \mathrm{L}$ (3). In proximal RTA, the amount of alkali required may be very large (up to $10-20 \mathrm{mmol} / \mathrm{kg} / \mathrm{day}$ ) due to the massive urinary bicarbonate loss (10). Conversely, distal RTA usually requires less sodium bicarbonate supply to be solved: (1-3 g/day) (3). Besides, it has been suggested that potassium citrate could be superior to sodium bicarbonate, because it lacks volume effects and obligatory calcium excretion associated with sodium (36). It has been documented that bone surface, connectivity density, cortical thickness, and cortical porosity are better preserved with potassium citrate than with potassium chloride (36-38). For instance, when a reversible RTA inducing mechanism is detected, one should try to remove the cause, as is the case of a drug-induced RTA or hyperkalemia-induced RTA by withdrawing or adjusting the drug dose or normalizing serum potassium levels, respectively (3). With respect to changing HMA inducing drugs, Schulte et al. have 
recently reported that a late conversion from a tacrolimus-based immunosuppression to belatacept was safe, effective and significantly improved renal function and acid-base balance, independently of glomerular filtration rate, in kidney transplant recipients (38). With respect to normalizing serum potassium levels, it can be achieved by different strategies, such as low potassium diet, mineralocorticoid (eg: fludrocortisone), cationic exchange, resins, etc. It is worth pointing out that mineralocorticoid should be used with precaution in these patients due to their potent sodium retention effect $(14,28,38,39)$. Therefore, based on the exposed above the following HMA therapeutic algorithm can be proposed (Figure 1).

\section{Conclusion:}

Renal tubular acidosis is a relatively frequent complication in kidney transplant recipient, which can be harmful, and should be adequately treated in order to avoid its renal and systemic adverse effects.

\section{Compliance with Ethical Standards:}

Conflict of Interest: All the authors declare that they have no conflict of interest. 
Table 1: Types of renal tubular acidosis in kidney transplant

\begin{tabular}{|c|c|}
\hline RENAL TUBULAR ACIDOSIS TYPES & PATHOPHYSIOLOGIC MECHANISMS \\
\hline $\begin{array}{l}\text { Distal RTA (type la or classic RTA, rate } \\
\text { limited distal RTA) }\end{array}$ & $\begin{array}{l}\text { interstitial damage secondary to transplant } \\
\text { rejection, calcineurin inhibitors, or } \\
\text { persistent inflammatory status. }\end{array}$ \\
\hline $\begin{array}{l}\text { Hyperkalemic distal RTA without } \\
\text { aldosterone deficiency (type Ib RTA or } \\
\text { voltage- dependent RTA) }\end{array}$ & obstructive uropathy \\
\hline $\begin{array}{l}\text { Proximal RTA (type II RTA or RTA } \\
\text { secondary to bicarbonate loss) }\end{array}$ & $\begin{array}{l}\text { calcineurin inhibitors, persisting } \\
\text { hyperparathyroidism, Fanconi syndrome } \\
\text { secondary to drugs or ischemia }\end{array}$ \\
\hline $\begin{array}{l}\text { Distal RTA with bicarbonate loss (type III or } \\
\text { mixed RTA) }\end{array}$ & renal ischemia \\
\hline $\begin{array}{l}\text { Hyperkalemic RTA associated to } \\
\text { aldosterone deficiency (type IV RTA) }\end{array}$ & $\begin{array}{l}\text { renin-angiotensin-aldosterone system } \\
\text { suppressing drugs, trimethoprim- } \\
\text { sulfamethoxazole, calcineurin inhibitors, } \\
\text { adrenal insufficiency or hyporeninemic } \\
\text { hypoaldosteronism secondary to diabetic, } \\
\text { lupic or acquired immunodeficiency } \\
\text { syndrome nephropathy, acute transplant } \\
\text { rejection }\end{array}$ \\
\hline
\end{tabular}


Figure 1: Hyperchloremic metabolic acidosis of renal origin (HMAR) in kidney transplant: Its evaluation and treatment

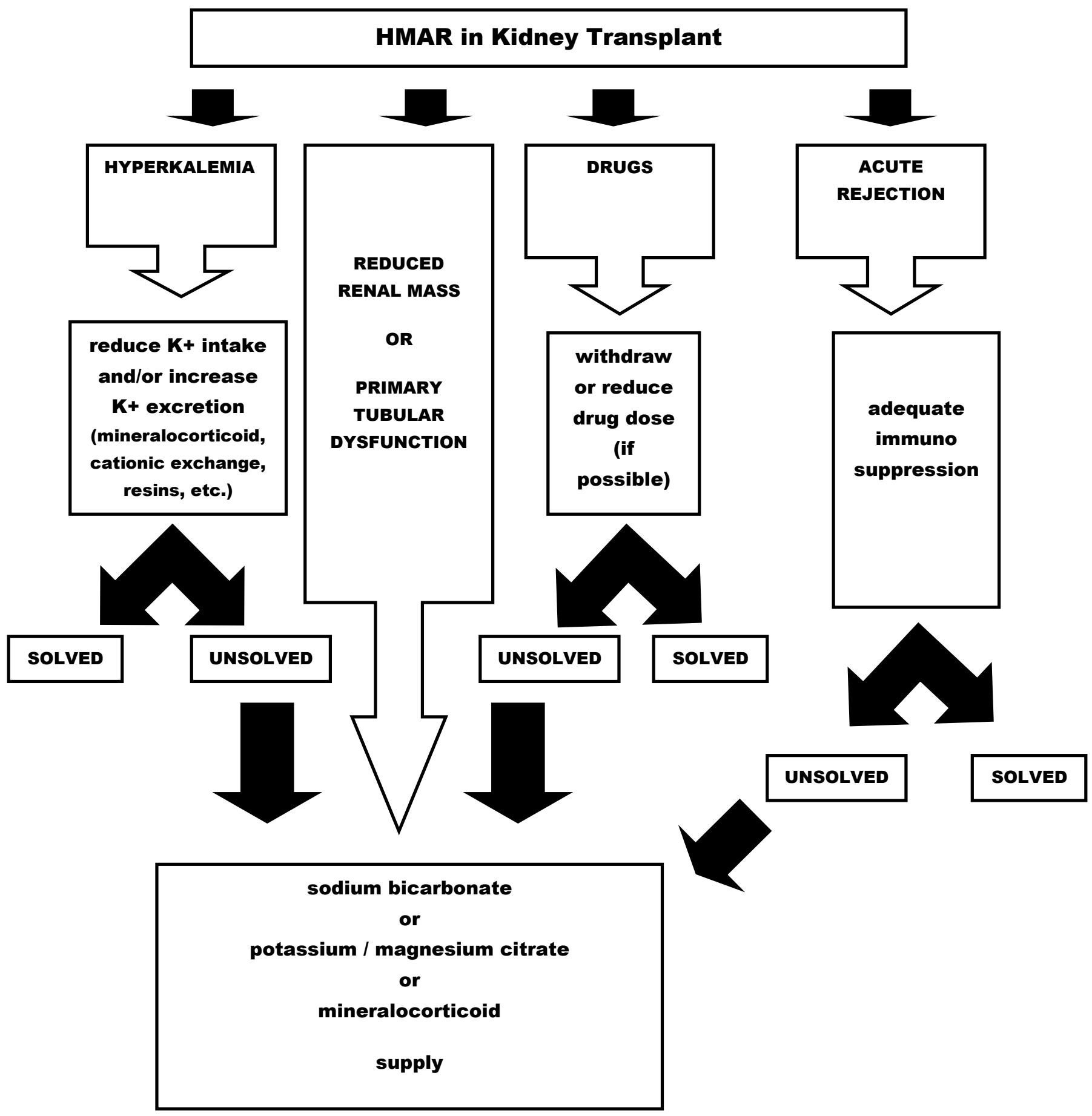




\section{References}

1) Georges B, Huart J, Krzesinski JM, Jouret F. [Screening and management of metabolic acidosis in patients with chronic kidney disease].Rev Med Suisse. 2018;14(615):14551458

2) Skiba K, Gojowy D, Szotowska M, Bartmańska M, Kolonko A, Cierpka L, Więcek A, Adamczak M.Metabolic acidosis in kidney transplant recipients. Pol Arch Intern Med. 2018 Oct 31;128(10):587-593. doi: 10.20452/pamw.4329.

3) Messa PG, Alfieri C, Vettoretti S.Metabolic acidosis in renal transplantation: neglected but of potential clinical relevance.Nephrol Dial Transplant. 2016;31(5):730-6. doi: 10.1093/ndt/gfv098.

4) Moorthi K, Batlle D. Renal tubular acidosis. In Gennari J, Adrogue H, Galla J, Madias N. (Eds.) Acid base disoreders and their treatments. Boca Raton. Taylor \& Francis. 2010: 273-304

5) Schwarz C, Benesch T, Kodras K, Oberbauer R, Haas M. Complete renal tubular acidosis late after kidney transplantation. Nephrology Dialysis Transplantation. 2006; 21: 2615-20.

6) Golembiewska E, Ciechanowski K.Renal tubular acidosis--underrated problem? Acta Biochim Pol. 2012;59(2):213-7.

7) Palazzo V, Provenzano A, Becherucci F, Sansavini G, Mazzinghi B, Orlandini V, Giunti L, Roperto RM, Pantaleo M, Artuso R, Andreucci E, Bargiacchi S, Traficante G, Stagi S, Murer L, Benetti E, Emma F, Giordano M, Rivieri F, Colussi G, Penco S, Manfredini E, Caruso MR, Garavelli L, Andrulli S, Vergine G, Miglietti N, Mancini E, Malaventura C, Percesepe A, Grosso E, Materassi M, Romagnani P, Giglio S. The genetic and clinical spectrum of a large cohort of patients with distal renal tubular acidosis. Kidney Int. 2017;91(5):1243-1255. doi: 10.1016/j.kint.2016.12.017.

8) Dagan A, Eisenstein B, Bar-Nathan N, Cleper R, Krause I, Smolkin V, Davidovits M. Tubular and glomerular function in children after renal transplantation. Pediatric Transplantation. 2005; 9: 440-44

9) Riveiro-Barciela M, Campos-Varela I, Tovar JL, Vargas V, Simón-Talero M, VenturaCots M, Crespo M, Bilbao I, Castells L. Hyperkalemic Distal Renal Tubular Acidosis Caused by Immunosuppressant Treatment with Tacrolimus in a Liver Transplant.Transplantation Proceedings. 2011; 43: 4016-18.

10) https://www.orpha.net/consor/cgi-bin/OC Exp.php?lng=ES\&Expert=47159

11) https://www.orpha.net/consor/cgi-bin/OC Exp.php?Lng=ES\&Expert=18 
12) Tanrisev M, Gungor $O$, Kocyigit, Kurtulmus $Y$,Tugmen $C$, Colak $H$, Altunoren $O$, Kebapci E, Karaca C. Renal Tubular Acidosis in Renal Transplant Patients: The Effect of Immunosuppressive Drugs. Annals of Transplantation. 2015; 20: 85-91.

13) Malik SI, Naqvi R, Ahmed E, Zafar MN. Prevalence and risk factors of renal tubular acidosis after kidney transplantation. Journal of Pakistan Medical Association. 2011; 61 (1): 23-27.

14) Keven K, Ozturk R, Sengul S, Kutlay S, Ergun I, Erturk S, Erbay B. Renal tubular acidosis after kidney transplantation-incidence, risk factors and clinical implications. Nephrology Dialysis Transplantation. 2007; 22: 906-10.

15) Rangel EB, Gomes SA, Machado PG, dos Santos BF, Pestana JO, Pacheco-Silva A, Heilberg IP.Severe hyperkalemic type 4 renal tubular acidosis after kidney transplantation: a case report.Transplant Proc. 2006;38(9):3112-5.

16) Musso CG.Potassium metabolism in patients with chronic kidney disease. Part II: patients on dialysis (stage 5). Int Urol Nephrol. 2004;36(3):469-72

17) Adamczak M, Masajtis-Zagajewska A, Mazanowska O, Madziarska K, Stompór T, Więcek A. Diagnosis and Treatment of Metabolic Acidosis in Patients with Chronic Kidney Disease - Position Statement of the Working Group of the Polish Society of Nephrology.Kidney Blood Press Res. 2018;43(3):959-969. doi: $10.1159 / 000490475$.

18) Ambühl PM. Posttransplant metabolic acidosis: a neglected factor in renal transplantation? Current Opinion in Nephrology and Hypertension. 2007; 16:379-87.

19) Yakupoglu HY, Corsenca $A$, Wahl $P$, Wüthrich RP, Ambühl PM. Posttransplant acidosis and associated disorders of mineral metabolism in patients with a renal graft.transplantation. 2007; 84(9):1151-57.

20) Kocyigit I, Unal A, Kavuncuoglu F, Sipahioglu MH, Tokgoz B, Oymak O, Utas C. Renal tubular acidosis in renal transplantation recipients. Renal Failure. 2010; 32: 687-90.

21) Katalinić L, Blaslov K, Đanić-Hadžibegović A, Gellineo L, Kes $P$, Jelaković $B$, BašićJukić N. Metabolic acidosis- An underestimated problem after kidney transplantation? Acta Clinica Croatica. 2015; 54:486-91.

22) Hjelmesaeth J, Hagen M, Hartmann A, Midtvedt $K$, Egeland $T$, Jenssen $T$. The impact of impaired insulin release and insulin resistance on glucose intolerance after renal transplantation. Clin Transplant. 2002 Dec;16(6):389-96.

23) Cosio FG, Pesavento TE, Osei K, Henry ML, Ferguson RM.Post-transplant diabetes mellitus: increasing incidence in renal allograft recipients transplanted in recent years.Kidney Int. 2001;59(2):732-7. 
24) Roth D, Milgrom M, Esquenazi V, Fuller L, Burke G, Miller J. Posttransplant hyperglycemia. Increased incidence in cyclosporine-treated renal allograft recipients. Transplantation. 1989;47(2):278-81.

25) Vincenti F, Friman $S$, Scheuermann E, Rostaing L, Jenssen T, Campistol JM, Uchida K, Pescovitz MD, Marchetti P, Tuncer M, Citterio F, Wiecek A, Chadban S, El-Shahawy M, Budde K, Goto N; DIRECT (Diabetes Incidence after Renal Transplantation: Neoral C Monitoring Versus Tacrolimus) Investigators. Results of an international, randomized trial comparing glucose metabolism disorders and outcome with cyclosporine versus tacrolimus. Am J Transplant. 2007 Jun;7(6):1506-14. Epub 2007 Mar 12. Erratum in: Am J Transplant. 2008 Jan;8(1):1. Am J Transplant. 2008 Apr;8(4):908

26) Heldal TF, Ueland T, Jenssen T, Hartmann A, Reisaeter AV, Aukrust $P$, Michelsen A, Åsberg A. Inflammatory and related biomarkers are associated with post-transplant diabetes mellitus in kidney recipients: a retrospective study. Transpl Int. 2018;31(5):510-519. doi: 10.1111/tri.13116.

27) Gyory AZ, Stewart JH, George CR, Tiller DJ, Edwards KD. Renal Tubular Acidosis, acidosis due to hyperkalemia, hypercalcemia, disordered citrate metabolism and other tubular dysfunctions following human renal transplantation. The Quarterly Journal of Medicine. 1969; 38(150):231-54

28) Harris AN, Grimm PR, Lee HW, Delpire E, Fang L, Verlander JW, Welling PA, Weiner ID. Mechanism of Hyperkalemia-Induced Metabolic Acidosis. J Am Soc Nephrol. 2018;29(5):1411-1425. doi: 10.1681/ASN.2017111163.

29) Adrogue HJ, Wesson DE. Acid-base. Houston. Gemini.1991

30) Blankenstein KI, Borschewski A, Labes R, Paliege A, Boldt C, McCormick JA, Ellison DH, Bader M, Bachmann S, Mutig K.Calcineurin inhibitor cyclosporine A activates renal $\mathrm{Na}-\mathrm{K}-\mathrm{Cl}$ cotransporters via local and systemic mechanisms. Am J Physiol Renal Physiol. 2017 Mar 1;312(3):F489-F501. doi:

10.1152/ajprenal.00575.2016.

31) Lin W, Mou L, Tu H, Zhu L, Wang J, Chen J, Hu Y. Clinical analysis of hyperkalemic renal tubular acidosis caused by calcineurin inhibitors in solid organ transplant recipients.J Clin Pharm Ther. 2017;42(1):122-124. doi: 10.1111/jcpt. 12485

32) Better OS, Chaimowitz C, Alroy GG, Sisman I. Spontaneous remission of the defect in urinary acidification after cadaver kidney homotransplantation. Lancet. 1970; 1(7638):110-2.

33) François $\mathrm{H}$, Mariette $X$.Renal involvement in primary Sjögren syndrome. Nat Rev Nephrol. 2016;12(2):82-93. doi: 10.1038/nrneph.2015.174.

34) Banhara PB, Gonçalves RT, Rocha PT, Delgado AG, Leite M Jr, Gomes CP. Tubular dysfunction in renal transplant patients using sirolimus or tacrolimus. Clinical Nephrology. 2015;63(6):331-7. 
35) Wiegand A, Ritter A, Graf N, Arampatzis S, Sidler D, Hadaya K, Müller TF, Wagner CA, Wüthrich RP, Mohebbi N. Preservation of kidney function in kidney transplant recipients by alkali therapy (Preserve-Transplant Study): rationale and study protocol. BMC Nephrol. 2018 Jul 13;19(1):177. doi:10.1186/ -018-0956-8.

36) Kulkarni M. Type 4 renal tubular acidosis in a kidney transplant recipient. Biomedical journal. 2016;39(1):85-6.

37) Starke A, Corsenca A, Kohler T, Knubben J, Kraenzlin M, Uebelhart D, Wüthrich $R P$, von Rechenberg $B$, Müller R, Ambühl PM.

Correction of metabolic acidosis with potassium citrate in renal transplant patients and its effect on bone quality. Clin J Am Soc Nephrol. 2012;7(9):1461-72

38) Ali SR, Shaheen I, Young D, Ramage I, Maxwell H, Hughes DA, Athavale D, Shaikh MG. Fludrocortisone-a treatment for tubulopathy post-paediatric renal transplantation: A national paediatric nephrology unit experience. Pediatr

Transplant. 2018 Mar;22(2). doi: 10.1111/petr.13134

39) Schulte K, Vollmer C, Klasen V, Bräsen JH, Püchel J, Borzikowsky C, Kunzendorf U, Feldkamp T. Late conversion from tacrolimus to a belatacept-based immunosuppression regime in kidney transplant recipients improves renal function, acid-base derangement and mineral-bone metabolism. J Nephrol. 2017Aug;30(4):607-615. doi: 10.1007/s40620-017-0411-0. 